2006-1265: IMPROVEMENT OF AN ELECTRICAL ENGINEERING COURSE OFFERED TO NON-ELECTRICAL ENGINEERING MAJORS

Qian Du, Mississippi State University

Judy Schneider, Mississippi State University 


\title{
Improvement of an Electrical Engineering Course Offered to Non-Electrical Engineering Majors
}

\begin{abstract}
ECE3183 Electrical Engineering Systems is a survey course offered by the Department of Electrical and Computer Engineering (ECE) to non-majors in all other engineering departments at Mississippi State University (MSU). A challenge in most service courses is how to motivate the students to learn. A primary reason that the students struggle in this class is that they do not understand the link between the knowledge in ECE3183 and their majors. To addressing this situation, a faculty member in the ECE department works closely with faculty in other engineering departments to incorporate major-related applications into lectures by the design of application-oriented examples. In this paper, we report an initial effort made by the ECE and Mechanical Engineering (ME) faculty to improve the teaching and learning effectiveness in ECE3183. The ME department was selected because they provide approximately $40 \%$ of the student population to ECE3183. Based on the experience and lessons learned from this initial effort, this concept will be extended to other departments in the near future.
\end{abstract}

\section{Introduction}

One key mission of baccalaureate engineering programs is to develop and offer the interdisciplinary coursework that is essential to preparing highly-qualified engineering graduates who will be successful and productive in their future careers. ${ }^{1}$ As Electrical Engineering (EE) has increasing cross-correlation with other engineering fields, most engineering institutions offer an introductory course in EE to non-electrical engineering (non-EE) students.

At Mississippi State University (MSU), the Department of Electrical and Computer Engineering (ECE) is tasked with offering such a "service" course, ECE3183 Electrical Engineering Systems, to non-EE majors in other engineering departments. In this course, basic circuit theories and analysis techniques are introduced, as well as some fundamental theories about electromagnetics and DC machines. This course is required for non-EE majors to fulfill the engineering science credits for their degree.

As in most service courses, how to motivate the students to learn is a challenge. Many students feel the only reason to take ECE3183 is to complete the course requirement for their degree, and possibly to prepare for the FE exam. A primary reason that the students struggle in this class is that they do not understand the link between the course content and their majors. The content of such an EE service course generally is a cut-and-paste combination of the course content offered to the EE students, which does not well correlate with the courses taken by non-EE majors. ${ }^{2}$ As a result, non-EE majors regularly express dissatisfaction and think it should no longer be required in their program of study. This type of feedback by students has also been expressed by many faculty members teaching a similar course at various universities. ${ }^{2}$

To addressing this situation, the ECE department works closely with faculty in other engineering departments to incorporate disciplinary application-oriented principles into the lectures. In this 
paper we report on an initial effort made by the ECE and Mechanical Engineering (ME) faculty to improve the teaching and learning effectiveness in ECE3183. The ME department was selected because they provide the largest student population to ECE3183 (40\% of the class). Based on the experience and lessons learned from this initial effort, this concept will be extended to other departments in the near future.

\section{Current ECE3183 at MSU}

ECE3183 at MSU consists of three hours of lecture with no supporting laboratory. The loss of a traditionally dedicated laboratory results from the reduction in credit hours to obtain a baccalaureate degree in the various engineering disciplines. Student population in ECE3183 includes, approximately, 40\% Mechanical Engineering, 30\% Chemical Engineering, 10\% Aerospace Engineering, 10\% Civil Engineering, and 10\% Industrial Engineering students. Around 70 students per semester take this course, which is offered 3 times per year.

The topics covered in ECE3183 are summarized in Table 1. The course objectives include: ${ }^{3}$

1) to develop the students' basic understanding of electric circuit analysis by the use of resistive networks;

2) to further develop the students' comprehension of basic circuits by extending the analysis techniques to AC circuit analysis; and

3 ) to introduce the students to the basics of electrical engineering.

Table 1: Topics covered in ECE3183 at MSU.

\begin{tabular}{|c|c|}
\hline Chapters & Topics \\
\hline \multirow{2}{*}{ 1. Circuit Fundamentals } & Circuit Elements and Laws \\
\hline & Series and Parallel Circuits \\
\hline \multirow{4}{*}{ 2. Resistive Circuits } & Node Voltage and Mesh Current Analysis \\
\hline & Thevenin and Norton Equivalent Circuit \\
\hline & Superposition \\
\hline & Wheatstone Bridge \\
\hline \multirow{4}{*}{ 3. AC Circuits } & Capacitance and Inductance \\
\hline & Phasor \\
\hline & Complex Impedance \\
\hline & AC circuit Analysis \\
\hline \multirow{3}{*}{ 4. Power } & AC Power Calculation \\
\hline & Power-Factor Correction \\
\hline & Balanced 3-phase System \\
\hline \multirow{3}{*}{ 5. Electromagnetics } & Magnetic Fields and Circuits \\
\hline & Inductance and Mutual Inductance \\
\hline & Ideal and Practical Transformer \\
\hline 6. Energy Conversion & Principles of DC Machines \\
\hline
\end{tabular}

Similar to the situation encountered at most other U. S. universities offering a similar course, the lecture did not provide clear interconnection between the course content and applications in different majors. The lack of connectivity is only worsened when there is no laboratory component to provide the students with hands-on experience. 


\section{Proposed Approach to the Coordination of ECE3183 and ME3701}

\subsection{ME3701 Experimental Orientation at MSU}

One of the major applications of electrical circuits in the ME curriculum was found to be in their laboratories. When conducting an experiment, the majority of the instrumentation and data recording relies on a knowledge of electrical circuits. The ME department at MSU offers a sequence of three laboratories, which are taken starting toward the end of the ME curriculum. It includes: ME3701 Experimental Orientation (EO), ME4721 Experimental Technique I (ET1), and ME4731 Experimental Technique II (ET2). The laboratories are intended to provide a hands-on environment to reinforce concepts learned in other courses, including those outside the ME home department. ME3701 (EO) gives the students an introduction to the use of instrumentation for basic measurements, the acquisition and processing of the measurement process. The second course in the sequence, ME4721 (ET1), concentrates on identification of the key parameters needed to guide the design of experiments using uncertainty analysis. These concepts are merged into ME4731 (ET2), which provides the opportunity for students to combine the knowledge gained in both EO and ET1 to plan an experiment which compares their theoretical predictions with the measured outcome. ${ }^{4}$

All these three laboratories, taken in sequence, are one credit each without an accompanying lecture. The first course, ME3701 focuses on measuring techniques and data acquisition. The recommended sequencing for ME3701 is the second semester of the junior year, which coincides with the sequencing of ECE3183. Since ECE3183 does not have a laboratory, it is being used as a complement to ME3701, and vice versa.

The primary objective of ME3701 is to cover three components: 1) fundamentals and use of transducers in mechanical engineering measurements, 2) computer-based data acquisition, and 3) an introduction to uncertainty analysis. Examples of typical experiments assigned in ME3701 include: 1) voltage and current measurements; 2) strain gauge analysis of a bending beam; 3 ) density determination using Archimedes Principle; 4) rotameter calibration; 5) resistance measurements utilizing LabView; 6) thermocouple time constant analysis; 7) vibrational mode analysis using an accelerometer mounted on a vibrating beam; 8) generate a stress vs. strain curve using an uniaxial tensile tester. ${ }^{5}$

\subsection{Coordination between ECE3183 and ME3701}

Because of the use of basic instrumentation in the first course in the ME laboratory sequence, ME3701, it is of the most interest for developing a collaboration with ECE3183. Due to the complimentary nature, the faculty members teaching ECE3183 and ME3701 work closely together to coordinate course schedule and develop examples and homework problems that can apply the knowledge in ECE3183 to the experiments in ME3701. Thus the experiments in ME3701 will motivate students enrolled in the classes of ECE3183 and help them relate to the concepts learned in ECE3183. The following are two examples which demonstrate the collaboration.

Example 1: Voltmeters and Ammeters 
Voltmeters and ammeters are briefly taught in ECE3183, but there is no laboratory provided for hands-on opportunity. Meanwhile, in ME3701, the students use voltmeters and ammeters to measure voltage and current in a circuit, but without the background discussion of the theory. The proposed coordination is presented as below.

a) In ME3701, the students were told that a voltmeter should have a parallel connection with the element whose voltage is to be measured, and an ammeter should have a series connection with the element whose current is to be measured. But they may not understand the reasons behind. In ECE3183, the basic structure of voltmeter and ammeter is introduced, which was not included in the previous course schedule. After being presented the internal structures of these instruments, students are able to clearly understand the usage of voltmeters and ammeters.

A primitive ammeter includes a current-carrying wire and a string in a magnetic field, as shown in Figure 1. ${ }^{6}$ According to the Lorentz's law, a force $F$ will be generated when a current $i$ to be measured flows through the wire, which equals

$$
F=B i L
$$

where $B$ is the magnetic flux density and $L$ is the length of the wire. This reduces the problem of measuring a current to the more familiar problem of measuring a force. With no current flowing through the wire, the spring is at its unstretched length. As the current $i$ flows through the wire, the spring stretches in a distance $x$ and develops the force $F_{x}$ required to balance the electromagnetic force $F$. Hence,

$$
K x=B i L
$$

where $K$ is the spring constant. This equation can be solved for the current:

$$
i=\frac{K x}{B L}
$$

This structure can explain why an ammeter is in series connection with an element whose current is to be measured. This also provides a good practical example to show the application of Lorentz's law in the lecture of electromagnetics in ECE3183, which the students find very difficult to understand.

A voltmeter may be constructed easily by modifying the basic ammeter as shown in Figure 2, where a large resistor is placed in series with the ammeter. When the instrument is connected to a voltage source, the current in the instrument is an indication of the voltage. This also explains why a voltmeter should be in parallel connection with an element whose current is to be measured.

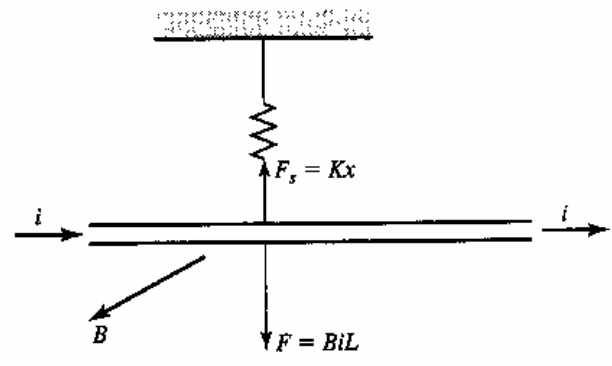

Figure 1: The primitive ammeter. ${ }^{6}$

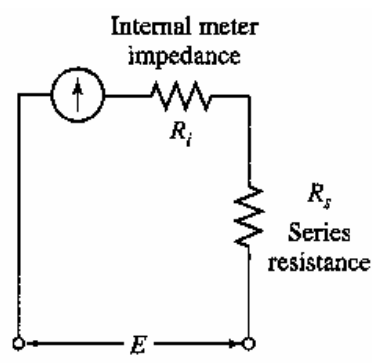

Figure 2: Voltmeter built from ammeter. ${ }^{6}$ 
b) When the voltage and current division principles are covered in ECE3183, the students know that an ideal ammeter should have zero resistance such that its existence does not change the current to be measured, while an ideal voltmeter should have infinitely large resistance such that its connection to the circuit does not change the voltage to be measured. However, in practice, no ammeter and voltmeter are ideal, so all measurements are imprecise.

In ME3701 error analysis experiment is designed to compare the theoretical and measured currents and voltages to further understand these concepts in ECE3183. Students are also taught how to use a non-ideal ammeter and voltmeter to retrieve the precise current and voltage in ME3701. Related homework problems are assigned and discussed in the classes of ECE3183.

\section{Example 2: Wheatstone Bridge}

A Wheatstone bridge covered in ECE3183 is normally used for the measurement of resistances in the range of $1 \Omega$ to $1 \mathrm{M} \Omega$. A schematic of the bridge is given in Figure 3. The bridge is called "balanced" when the potential difference points B and D is zero, which can be sensed by closing the switch $\mathrm{S}_{2}$ and adjusting the value of $R_{1}$ until the sensing device indicates no current flow. When the bridge is balanced,

$$
i_{1}=i_{x}=\frac{E}{R_{1}+R_{x}}, i_{2}=i_{3}=\frac{E}{R_{2}+R_{3}} .
$$

Then the unknown resistance $R_{x}$ can be determined by

$$
R_{x}=\frac{R_{1} R_{3}}{R_{2}} .
$$

If accurate measurements are to be made with a bridge circuit, the values of the resistors $R_{1}, R_{2}$, and $R_{3}$ must be precisely known, and the sensor should be sufficiently sensitive to detect small degrees of imbalance.

In ME3701, the use of a Wheatstone bridge is programmed into LabView. To help the students understand the Wheatstone bridge, an experimental problem is designed to use a Wheatstone bridge for resistance measurement. Results from the Wheatstone bridge input into LabView are compared and the "rules of thumb" about resistor selection in a Wheatstone bridge can be derived.

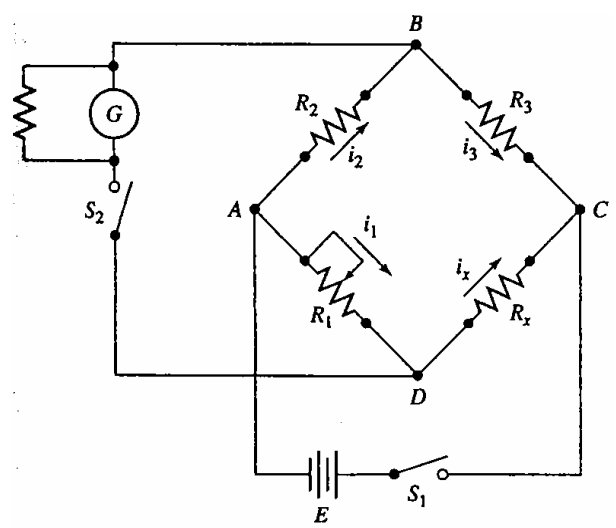

Figure 3: Schematic of basic Wheatstone bridge. ${ }^{6}$ 
This provides a good background in the use of a Wheatstone bridge to record strain measurements. A strain gauge mounted on a uniaxial tension specimen, is used as input to one leg of the Wheatstone bridge. As the specimen is strained, the corresponding change in resistance provides an imbalance to the Wheatstone bridge, which can be used to determine the amount of strain experimented by the specimen under load. Again by coordinating lectures, the application of a Wheatstone bridge to the strain measurements in the ME3701 laboratory is first discussed in the lecture of ECE3183 to cover the theory, and then reinforced in the laboratory as the students measure strain by use of a strain gauge mounted on a uniaxially strained specimen.

\subsection{Practical Considerations}

Although the coordinated lectures and homework problems are initially designed for ME majors at this current stage, other majors can also benefit. Because the fundamental concepts taught in ECE3183 are better understood with practical hands-on applications, and the classes are made more interesting. Moreover, the knowledge in ECE3183, such as electric circuits, is useful to the basic measurements in experiments made by all majors.

In order for all the students in ECE3183 to have hands-on experience, hardware homework will be assigned. An Electronics Learning Lab box will be purchased for each group with three to four students, which includes: all necessary parts and wires to build projects designed to employ concepts in ECE3183, manuals for project instruction, solderless breadbord for easy circuit construction, and build-in analog meters for quick result measurement. This will be a great remedy of the loss of a traditional supporting lab.

A majority of ME students will take ECE3183 and ME3701 in the same semester in their junior year. As a part of coordination plan, the ME department will strongly encourage their students to take these two required courses simultaneously. It is possible for a small number of students (e.g., transfer students) to have to postpone the completion of ECE3183 until their senior year. Since these students have taken ME3701 before, the improvement of ECE3183 will help them make a clearer link with what they have done in ME3701. The hardware homework assignments will give them a second hands-on opportunity. No student takes ECE3183 earlier than ME3701.

\subsection{Assessment Plan}

The performance of all the students and ME majors in both ECE3183 and ME3701 will be carefully evaluated and analyzed. Our assessment plan includes three components: 1) assessment among the ME students in ECE3183,2) assessment among all students taking ECE3183, and 3) assessment among all students finishing ECE3183.

\section{Assessment Methodology}

As we have known, assessment methodologies can be divided into two primary types: studies that describe the current state of a phenomenon, referred to as descriptive studies; and studies that examine how a phenomenon changes as a result of an intervention, referred to as experimental studies. ${ }^{7}$ In our assessment, the descriptive studies will be adopted. This is because we lack previous quantitative data as a baseline for the experimental studies. When implementing the descriptive studies, Mixed research methods (qualitative and quantitative) will 
be used, where surveys and interviews will be carefully designed.$^{8-9}$ Both open-ended and selected-response questions will appear on self-report surveys to maximize the information to be acquired: open-ended questions are analyzed qualitatively providing the students opportunities to express their opinion in an unstructured format, while selected-response questions give us quantitative desired information.

\section{Assessment among ME Students in ECE3183}

A mid-term survey as well as an end-semester survey will be conducted. Possible selectedresponse (Strongly Agree, Agree, Neutral, Disagree, Strongly Disagree) questions include "ME3701 helps me understand the concepts in resistive circuits", "ME3701 helps me understand the concepts in electromagnetics", "the lectures in ECE3183 helps me conduct experiments in voltage and current measurement", "the lectures in ECE3183 helps me conduct LabView experiments in resistance measurement", "the lectures in ECE3183 helps me conduct strain gauge analysis", "the schedule coordination between ECE3183 and ME3701 is reasonable". Possible open-ended questions are "any other help that I get from the coordination of ECE3183 and ME3701", "suggestions for improvement".

For a small number of ME students who have taken ME3701 before, they will only participate in the exit interview as a group. The purpose of such an in-person interview is to collect the most authentic data that reflects the student perspective about the class. Because it is quite timeconsuming, the subjects include 3-5 students being at the similar grade. Students belonging to different grade groups will be interviewed separately to avoid any potential conflict. The semistructured interview will be designed, where a predetermined set of questions is to be followed by probing questions to better understand the interviewed students' response.

\section{Assessment among All the Students Taking ECE3183}

An assessment of hardware homework assignment with selected-response and open-ended questions can be accomplished by a questionnaire, which is required to complete at the conclusion of each hardware assignment. In this questionnaire, five to six questions are designed to get desired information, such as "the assignment helps me a better understanding of concepts taught in class", "the assignment takes a reasonable time". Two to three essay questions are for the acquisition of additional information, such as "things I like about this assignment", "suggestions for improvement".

\section{Assessment among All the Students Finishing ECE3183}

In each engineering department, evaluation among graduating seniors is conducted to know their opinion about their undergraduate program. Among all the selected-response questions in the Graduating Senior Survey, a question about ECE3183 will be added, such as "apply my knowledge of ECE3183 appropriately to my major". Percentage of answers of "Strongly Agree" and "Agree" will be collected.

The FE exam (general) result will also be considered. ${ }^{10-11}$ Based on the percentages of correctly answered questions in the area of Electricity and Magnetism, the performance of our students will be compared against the peer performance to gauge the effectiveness of ECE3183. 


\section{Conclusion}

To improve the teaching and learning effectiveness of the service course, ECE 3183 at MSU, initial efforts have been made to coordinate the lectures offered by the ECE department with the laboratory offered by the ME department. We believe that this collaboration will inject vigor into what has historically been perceived as a "boring" classroom in the ECE3183 course, while enhancing the ME curriculum.

If the proposed integration is proved to be successful, a similar approach will be applied to the Chemical Engineering majors, since their population is the second largest, followed by Civil Engineering, Aerospace Engineering, and Industrial Engineering. Examples related to different majors will be discussed in class, and different application-oriented homework problems may be assigned to different majors.

We also believe that this experience will be instructive to the improvement of similar courses offered at other universities.

\section{Reference}

1. Bordogna, J., Fromm, E., and Ernst, E. W., "Engineering education: innovation through integration," Journal of Engineering Education, vol. 82, no. 1, pp. 3-8, 1993.

2. S. A. Zekavat, C. Sandu, G. Archer, and K. Hungwe, "An evaluation of the teaching approach for the interdisciplinary course electrical engineering for non majors," Proceedings of 2005 ASEE conference, Portland, Oregon, June 2005.

3. Hambley, A. R., Electrical Engineering: Principles and Applications, 3rd Edition, Prentice-Hall, 2002.

4. Hudson, S. T., Schneider, J. A., and Steele, W. G., "Uncertainty analysis for undergraduate and graduate Mississippi State University Mechanical Engineering Students," Proceedings of 2003 AIAA conference.

5. Steele, W. G., and Schneider, J. A., "Experiences in an undergraduate laboratory using uncertainty analysis to validate engineering models with experimental data," Proceedings of 2005 ASEE conference, Portland, Oregon, June 2005.

6. Holman, J. P., Experimental Methods for Engineers, 7th Edition, McGraw-Hill, 2001.

7. B. M. Olds, B. M. Moskal, and R. L. Miller, "Assessment in engineering education: evolution, approaches and future collaboration,"Journal of Engineering Education, vol. 94, no.1, pp.13-25, 2005.

8. L. A. Suskie, Questionnaire Survey Research: What Works, $2^{\text {nd }}$, Florida State University: Association for Institutional Research, 1996.

9. J. A. Leydens, B. M. Moskal, and M. J. Pavelich, "Qualitative methods used in the assessment of engineering education," Journal of Engineering Education, vol. 93, no. 1, pp. 65-72, 2004.

10. J. L. Waston, "An analysis of the value of the FE examination for the assessment of student learning in engineering and science topics," Journal of Engineering Education, vol. 87, no. 3, pp. 305-320, 1998.

11. N. Nirmalakhandan, D. Daniel, and K. White, "Use of subject-specific FE exam results in outcomes assessment," Journal of Engineering Education, vol. 93, no. 1, pp. 73-77, 2004. 